Doug Geisler, Eva K. Grebel, and Dante Minniti, eds.

\title{
Cluster Mass and Metallicity Distributions: Reconstructing the Events During Halo Formation
}

\author{
William E. Harris \\ Department of Physics and Astronomy, McMaster University \\ Hamilton L8S $4 M 1$ Canada
}

\begin{abstract}
Globular clusters in most large galaxies are a mixture of metal-poor and metal-rich (bimodal), but the halo stars are almost entirely metal-rich. This and other lines of evidence argue that the metalpoor globular clusters formed within widely distributed $10^{8}-10^{9} M_{\odot}$ gas clouds (supergiant GMCs) during an early burst in which most of the gas was ejected or unused till later rounds of star formation.

New simulations of the growth of pre-galactic potential wells in the early universe now indicate that the initial power-law form of the globular cluster mass distribution $\left(d N / d M \sim M^{-1.8}\right)$ is a miniature replica of the mass distribution of the SGMCs themselves, which grow hierarchically in the CDM potential wells of large protogalaxies.
\end{abstract}

\section{Introduction}

The simple goal of employing globular clusters as archaeological clues to galaxy formation has become increasingly complicated over the past decade by the growing evidence that globular cluster systems (GCSs) might have been strongly influenced by major mergers and ongoing accretion events. Decoding the meaning of the cluster metallicity distribution function (MDF) or the luminosity distribution function (LDF or GCLF) has become more ambiguous.

The issue is made still more difficult by the realization that the GCS is a highly biased and tiny subsample of the old-halo population: the host galaxy is, by definition, made up of the field-halo stars, of which perhaps $0.3 \%$ find themselves in globular clusters at present (McLaughlin 1999). How typical is the MDF of the clusters (e.g.) of the halo as a whole? By studying the GCS in detail, are we actually learning more about globular cluster formation processes than about the larger problem of galaxy formation?

\section{NGC 5128: A Case Study in MDFs}

Except for the Milky Way and a few galaxies in the Local Group, it has not been possible to compare MDFs of the globular clusters and halo stars within the same galaxy in detail till recently; and till now, the important gE type of galaxy has been out of reach. We are just beginning to take some important steps in this direction. 


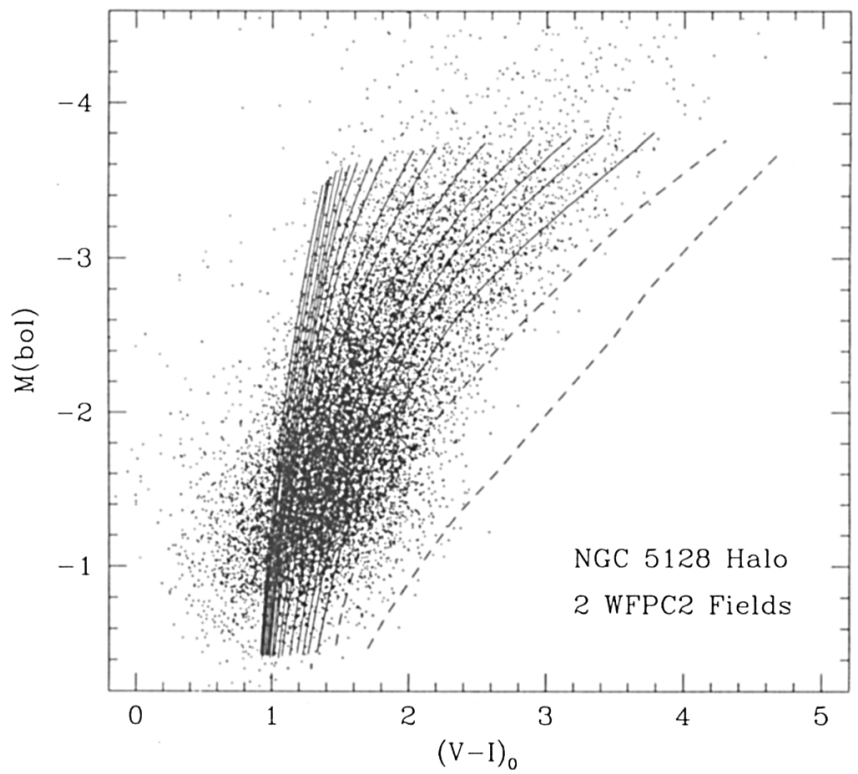

Figure 1. Color-magnitude array for the NGC 5128 halo stars in the 21-kpc and 31-kpc target fields, from Harris \& Harris (2000). The red-giant model tracks extending up to the helium flash extend from heavy-element abundances of $0.005 Z_{\odot}$ up to almost $3 Z_{\odot}$.

NGC 5128, easily the closest giant E galaxy at $d \sim 4 \mathrm{Mpc}$, is beginning to emerge as a keystone object. A series of photometric studies (Soria et al. 1996; G. Harris et al. 1999; Harris \& Harris 2000, 2001; Marleau et al. 2000) now give us a first deep look into the true composition of a giant elliptical on a direct, star-by-star basis. Harris et al. $(1999,2000,2001)$ give the results of deep $H S T /$ WFPC2 $(V, I)$ photometry in three fields at projected radial distances from $8 \mathrm{kpc}$ out to $31 \mathrm{kpc}$ from galaxy center. The color-magnitude diagrams of these "pencil-beam" samples of the halo reveal that (a) more than $99 \%$ of the stars can be interpreted as classically "old" red giants, with little or no contribution from younger AGB-type stars; and (b) their color distribution is enormously broader than can be explained by either photometric measurement scatter or internal age spread, strongly indicating that a large star-to-star metallicity spread is present.

A dense grid of red-giant evolutionary tracks (VandenBerg et al. 2000; Bertelli et al. 1994; see Harris \& Harris 2000 for detailed discussion and methodology) can be superimposed on the color-magnitude diagram of the halo stars to transform color $(V-I)_{0}$ into heavy-element abundance $Z$ and derive a first-order MDF (see Figure 1).

Most of the weight in the resulting MDF comes from the brighter stars in the diagram $\left(M_{b o l} \lesssim-2.5\right)$, where photometric scatter is minimal and the model track separation is largest. The overwhelming majority of the stellar population even in the outer halo falls in the range $-1<\log \left(Z / Z_{\odot}\right)<0$. There are almost no metal-poor stars, nor are there many with above-Solar metallicities. Similarly, small fractions of low-metallicity stars have been deduced for other $\mathrm{E}$ 
galaxies from population synthesis of their integrated light (e.g., Lotz et al. 2000; Maraston \& Thomas 2000), suggesting that what we see directly in NGC 5128 may be a general pattern for ellipticals.

In rough terms, what does this mean for the evolutionary history of the galaxy? A standard closed-box Simple Model of chemical evolution (which gives $d n / d Z \sim \exp (-Z / y)$ for an initial supply of primordial $Z=0$ gas and where $y$ is the effective nucleosynthetic yield) does well at matching the upper end of the $\operatorname{MDF}\left(Z \gtrsim Z_{\odot} / 3\right)$ but does very poorly at the low end. Such a model strongly overpredicts the relative numbers of low $-Z$ stars in NGC 5128 .

Within the context of these types of broad-brush models, the most plausible alternative explanations are (a) adopting a different IMF at early times, more strongly weighted to high-mass stars, or (b) allowing continued infall of unenriched gas during the first phases of star formation (an "accreting-box" model; see Binney \& Merrifield 1998).

The variable-IMF option cannot be definitively ruled out, but is an arbitrary measure for which there is little or no compelling evidence from the wide variety of present-day star-forming regions we see around us now. By contrast, option (b) - ongoing gas accretion during the rapid early phases of star formation - is something that we would expect to happen anyway for giant ellipticals at the centers of groups of galaxies, regardless of other factors.

Once the infall option is introduced, we need to specify the rate at which it happens. Harris \& Harris (2001) define a simple "delayed exponential" infall model in which the gas accretion rate $d M / d t$ is assumed to be constant for some initial time interval $\tau_{1}$ and then declines exponentially over an $e$-folding time $\tau_{2}$. Both the initial infall rate and the fiducial times $\tau_{1}, \tau_{2}$ are free parameters to be determined by the fit to the data.

Figure 2 displays a sample fit of one of these "accreting-box" models to the observed MDF for the outer-halo fields. The particular example shown has $\tau_{1}=0$, i.e. the gas accretion rate declines exponentially right from the start. The $e$-folding time $\tau_{2}$ here is set equal to 30 timesteps where in our numerical model, $5 \%$ of the ambient gas is converted to stars in each timestep $\delta t$. A plausible and conservative assumption is that most of the accretion takes place over the first $\sim 3 \mathrm{Gyr}$, after which the gas composition has built up to near-Solar levels. Under these circumstances our average enrichment timestep $\delta t$ would be about $30 \mathrm{Myr}$ and the initial accretion rates (and gas conversion rates) in the proto-elliptical are near $100 M_{\odot}$ per year.

Almost any giant elliptical should be expected to grow, not just by the direct conversion of gas to stars within its own potential well, but by the later (and ongoing) accretion of small satellites (e.g., Côté et al. 2000). How important is this accretion in actuality? Most stars in dwarf $\mathrm{E}$ galaxies, for example, have metallicities in the range $[\mathrm{m} / \mathrm{H}]=\log \left(Z / Z_{\odot}\right)<-0.7$. The extreme lack of such low $-Z$ stars in NGC 5128 therefore places rather stringent constraints on the numbers of dwarf ellipticals that could have been accreted. It appears necessary to conclude that the bulk of NGC 5128 was formed in situ in the classical way, or by mergers of much larger protogalactic clumps which could hold on to most of their gas and thus build upward to higher metallicity through continuing star formation and enrichment. 


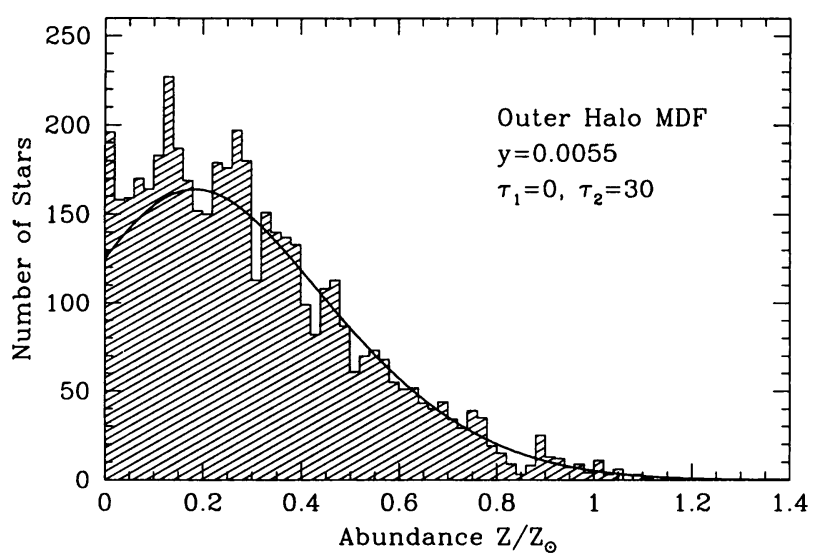

Figure 2. Metallicity distribution function (MDF) for the outer halo stars in NGC 5128, from Harris \& Harris (2000). Note that the MDF is plotted in its linear form as number of stars per unit heavy-element abundance $d n / d Z$. The solid line shows a model of chemical evolution starting with $Z=0$ gas and allowing gas infall at an exponentially decaying rate (see text).

\section{Globular Clusters versus Field Stars}

What of the globular clusters? Recent studies (G. Harris et al. 1992; Rejkuba 2001; Peng 2001) show repeatedly that the MDF for the NGC 5128 clusters (plotted now in its logarithmic version as number per unit $[\mathrm{m} / \mathrm{H}]$ ) has the bimodal form now well established as the norm for giant ellipticals: roughly equal numbers of clusters in metal-poor and metal-rich modes, centered approximately at $\log \left(Z / Z_{\odot}\right) \sim-1.4$ and -0.4 . These are shown in Figure 3.

The high-metallicity peak for the clusters matches up reasonably well with the peak of the stellar MDF at $\log \left(Z / Z_{\odot}\right) \simeq-0.4$, but the two distributions are strikingly different in their proportions of low-metallicity objects. It is just as obvious that the field stars, not the clusters, represent the MDF for the whole galaxy and are the primary indicator of its overall star forming history. Unless we can decipher the reason why the two distributions are different, it casts considerable doubt on our perhaps-optimistic claims from past years that the cluster MDF is a direct indicator of the galaxy formation process.

Having the two full distribution functions in hand for the first time within a giant elliptical allows us to construct a new kind of graph: the specific frequency (number of clusters per unit halo light) as a function of metallicity. This function is shown in Figure 4. Whereas $S_{N} \sim 2-3$ for the metal-richer part of the MDF (quite similar to the global average over all metallicities, since the vast majority of the light comes from the high $-Z$ stars), it is in the range $S_{N} \sim 10-20$ for the metal-poor component $([\mathrm{m} / \mathrm{H}]<-1)$. In fact, $S_{N}$ at the low $-Z$ end is even higher than shown here, because photometric scatter causes us to overestimate the numbers of stars in that part of the CMD (Fig. 1). There is thus an order 


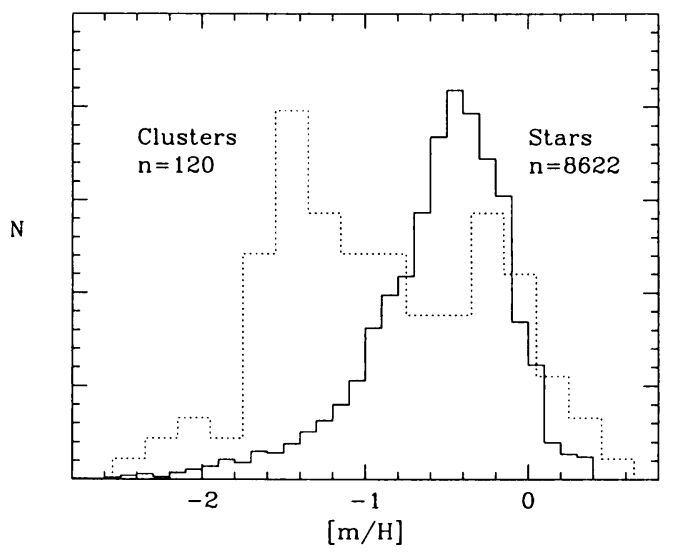

Figure 3. Metallicity distribution functions for the globular clusters and halo field stars in NGC 5128. The clusters (dotted line; from the combined data of G. Harris et al. 1992 and Rejkuba 2001) have the characteristic bimodal form seen in most giant ellipticals. The stars belong almost entirely to the metal-rich "mode".

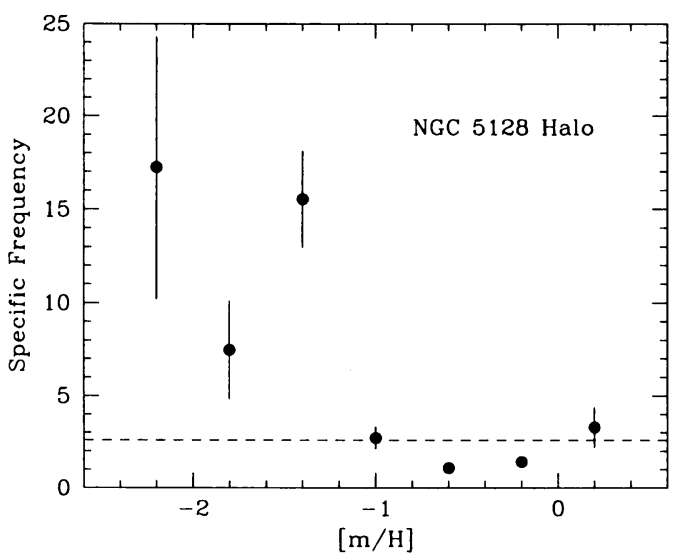

Figure 4. Specific frequency versus metallicity for the halo of NGC 5128 (from Harris \& Harris 2001). The total $S_{N}$ over the whole galaxy (dashed line) is at $S_{N}=2.6$. 
of magnitude difference between the metal-poor and metal-rich components in the "efficiency" with which they generated, or acquired, globular clusters.

Values of $S_{N} \gtrsim 20$ are almost unprecedented. The only previously known sites with such high ratios are a few of the smallest nucleated-dwarf ellipticals (McLaughlin 1999; W. Harris et al. 1998; W. Harris 2001). A plausible interpretation of such objects (Durrell et al. 1996; W. Harris et al. 1998; McLaughlin 1999) is that they began their initial starburst with the globular clusters forming earliest in the burst from the highest-density clumps. Then, the stellar winds and supernovae from these first few objects drove out most of the gas from its tiny potential well before it could be converted to stars. The net result was to leave behind a dE galaxy with only low-metallicity stars and an abnormally large number of clusters.

If something similar happened at early times in the NGC 5128 halo, then there might be two possible answers to the $S_{N}(Z)$ trend shown in Fig. 5:

- The halo stars with $[\mathrm{m} / \mathrm{H}] \lesssim-1$ were essentially all acquired by accretion of dE's, along with their (relatively numerous) metal-poor globular clusters. However, two worries are that (a) the observed $S_{N} \sim 20$ is an upper limit for known dE's, whereas it is an average for the metal-poor halo in NGC 5128. To make this work would require all the accreted satellites to be the very smallest types, which seems implausible, or else require that proto-dE's starting out within much larger potential wells behaved differently from isolated ones.

- Alternately, we might suppose that the first round of star formation in NGC 5128 took place in many widely distributed dwarf-sized clumps, each of which was located within the larger proto-gE potential well. These clumps were already busy forming stars as they were simultaneously falling together to assemble the eventual giant galaxy. Each clump could have acted locally like a $\mathrm{dE}$ as described above, with a short intense burst of star and cluster formation and ejection of most of the gas into the larger potential well of the proto-gE. This gas later recollected, initiating a second and more major round of star formation that built the bulk of today's visible $\mathrm{gE}$. The postulated gas clumps are the supergiant molecular clouds or SGMCs of Harris \& Pudritz (1994), or the dwarf-sized hosts of Burgarella et al. (2001). However, for this scenario to work, we must require globular cluster formation to occur early in any starburst.

\section{Mass Distribution Functions: Fact and Legend}

Much recent interest has centered on the mass distributions of the old globular clusters and those of young clusters such as the objects now known to be formed in starbursts in large numbers. The globular cluster mass distribution (or equivalently the luminosity distribution or LDF) is by tradition plotted as number of objects per unit magnitude (or log mass). In this plane it is roughly Gaussian in form, and the growing body of LDF observations continues to show how remarkably similar it is from one galaxy to another. By unfortunate contrast, the LDF for young clusters is often plotted in the more physically oriented plane of number per unit luminosity (or mass), where it appears more or less like a power law. The two planes are directly related $\left(d N / d m_{V} \sim d N / d \log M \sim M \cdot d N / d M\right.$ for magnitude $m_{V}$ and mass $M$ ), but an astonishing amount of discussion in 


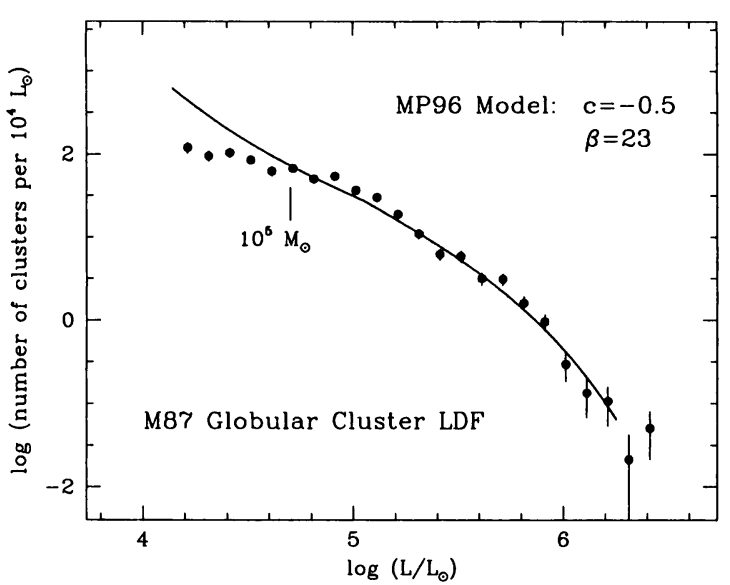

Figure 5. Luminosity distribution function (LDF) for globular clusters in M87 (data adapted from Kundu et al. 1999). Note here that the LDF is plotted in its linear form as number of clusters per unit $10^{4} L_{\odot}$. The solid line is a theoretical mass spectrum (McLaughlin \& Pudritz 1996). The specific model shown is for a timescale ratio $\beta=23$ (star formation time divided by cloud collision time) and for a cloud lifetime varying as $\tau \sim M^{-1 / 2}$.

the recent literature has emphasized the "differences" between old and young clusters which in fact boil down to this trivial issue of notation.

In the linear plane $(d N / d M$ or its observable surrogate $d N / d L)$, a typical LDF for old-halo clusters looks like the one shown in Figure 5. The particular case shown is for the prototypical giant elliptical M87. Above the $\sim 10^{5} M_{\odot}$ point which marks the traditional GCLF "turnover", a single power-law slope $d N / d L \sim L^{-1.8 \pm 0.2}$ is consistently found to match the GCSs in most or all galaxies. For young-cluster systems (the best-studied example being the Antennae clusters; Whitmore et al. 1999) the same power law slope is seen. Dynamical evolution over the first $1-2$ Gigayears has very likely been responsible for carving away the low-mass end $\left(<10^{5} M_{\odot}\right)$, but the higher-mass end maintains something close to its original shape (cf. Vesperini 2001; Fall 2001).

But there is one important contrast between the Antennae and M87 clusters which has not drawn enough attention. Their median masses are quite different. The Antennae merger is forming star clusters at a characteristic mass scale about one order of magnitude smaller than the traditional globular clusters in M87 and elsewhere. The Antennae merger certainly contains a few of the ultra-large GMCs of $\gtrsim 10^{8} M_{\odot}$ that we are looking for (Wilson et al. 2000), but the vast majority of its star-forming sites are within much smaller clouds which will not leave behind massive star clusters. The suggestion hidden in this comparison is that giant ellipticals like M87 formed by the assembly of GMCs that were, on average, much larger than we now see in the Antennae. It is all the more remarkable that the power-law shape of the mass distribution seems to be, roughly at least, independent of mass scale. 
Various plausible alternatives have been suggested for building the basic form of the initial mass distribution function, ranging from collisional growth of cloudlets within GMCs (McLaughlin \& Pudritz 1996) to turbulence spectra (Elmegreen \& Efremov 1997). It is worth emphasizing that the collisional-growth model can provide quantitative matches to the observed mass distributions in a natural way as long as the cloud lifetime against internal star formation is about an order of magnitude longer than the cloud/cloud collision time (see Harris \& Pudritz 1994; McLaughlin \& Pudritz 1996 for thorough discussion). Fortunately, this requirement is likely to be automatically true if turbulence and magnetic field are the dominant sources of internal pressure.

I will close this review with a few remarks on the role of the GMCs within which stars and star clusters form. Harris \& Pudritz (1994) postulated that reservoirs of gas $10^{8}-10^{9} M_{\odot}$ big - "supergiant" molecular clouds or SGMCs would be needed in order to build globular-cluster-sized protoclusters of $10^{5}$ $10^{6} M_{\odot}$, arguing from the empirical evidence that the biggest star clusters formed in a given GMC attain only a few thousandths of the whole GMC mass. Clearly, the very high star formation efficiency $e \sim 0.5$ necessary to form a gravitationally bound cluster is a rather rare occurrence. The emergent mass spectrum of their embedded star clusters would, in this scenario, be a miniature replica of the host GMC mass distribution if the characteristic ratio of protocluster mass to parent cloud mass $\eta \equiv M_{c l} / M_{G M C}$ is independent of mass scale. Lastly, the total number of clusters, and hence the specific frequency, is set by the number of clusters a given GMC can produce; again, strictly empirical evidence suggests that half a dozen bound clusters per GMC is typical.

Current work is filling in more of the comprehensive picture. Weil \& $\mathrm{Pu}$ dritz (2001) use N-body modelling within a cosmological CDM simulation to explicitly follow the buildup of gas clouds within the dark-matter potential well of a protogalaxy. They find that hierarchical growth creates a GMC cloud mass spectrum of the form $d N / d M \sim M^{-1.7 \pm 0.1}$. Furthermore, the "top end" of the mass distribution reaches $10^{9} M_{\odot}$ quite quickly (by redshift $z \simeq 3.5$ ). These features strongly resemble the dwarf-sized SGMCs that we have argued must be the necessary host sites for globular cluster formation in the early galaxy. They have also been cross-identified with the damped Ly $\alpha$ systems by Burgarella et al. (2001; see also Kissler-Patig 2001).

These simulations are both intriguing and encouraging, but we are still far from tying together the complete story. For example, the Weil/Pudritz models trace only what happens to the gas (the prescriptions for star formation are deliberately switched off); and we do not understand the crucial mass ratio parameter $\eta \sim 10^{-3}$ which sets the mass scale of the clusters within a given GMC. Nevertheless, there are grounds for genuine optimism that we are beginning to piece together a more comprehensive history for these fascinating objects.

\section{References}

Bertelli, G., Bressan, A., Chiosi, C., Fagotto, F., \& Nasi, E. 1994, A\&AS, 106, 275

Binney, J., \& Merrifield, M. 1998, Galactic Astronomy (Princeton University Press) 
Burgarella, D., Kissler-Patig, M., \& Buat, V. 2001, AJ, 121, 2647

Côté, P., Marzke, R. O., West, M. J., \& Minniti, D. 2000, ApJ, 583, 869

Durrell, P. R., Harris, W. E., Geisler, D., \& Pudritz, R. E. 1996, AJ, 112, 972

Elmegreen, B. G., \& Efremov, Y. 1997, ApJ, 480, 235

Fall, S. M. 2002, this conference

Harris, G. L. H., Geisler, D., Harris, H. C., \& Hesser, J. E. 1992, AJ, 104, 613

Harris, G. L. H., \& Harris, W. E. 2000, AJ,120, 2423

Harris, G. L. H., Harris, W. E., \& Poole, G. B. 1999, AJ, 117, 855

Harris, W. E. 1991, ARA\&A, 29, 543

Harris, W. E. 2001, in Star Clusters, Saas-Fee Advanced Course 28, Swiss Society for Astrophysics and Astronomy, edited by L. Labhardt \& B. Binggeli (New York: Springer)

Harris, W. E., \& Harris, G. L. H. 2001, in preparation

Harris, W. E., Harris, G. L. H., \& McLaughlin, D. E. 1998, AJ, 115, 1801

Harris, W. E., \& Pudritz, R. E. 1994, ApJ, 429, 177

Kissler-Patig, M. 2002, this conference

Kundu, A., Whitmore, B. C., Sparks, W. B., Macchetto, F. D., Zepf, S. E., \& Ashman, K. M. 1999, ApJ, 513, 733

Lotz, J. M., Ferguson, H. C., \& Bohlin, R. C. 2000, ApJ, 532, 830

Maraston, C., \& Thomas, D. 2000, ApJ, 541, 126

Marleau, F. R., Graham, J. R., Liu, M. C., \& Charlot, S. 2000, AJ, 120, 1779

McLaughlin, D. E. 1999, AJ, 117, 2398

McLaughlin, D. E., \& Pudritz, R. E. 1996, ApJ, 457, 578

Peng, E. 2002, this conference

Rejkuba, M. 2001, AAp, 369, 812

VandenBerg, D. A., Swenson, F. J., Rogers, F. J., Iglesias, C. A., \& Alexander, D. R. 2000, ApJ, 532, 430

Vesperini, E. 2002, this conference

Soria, R. et al., 1996, ApJ, 465, 79

Weil, M. L., \& Pudritz, R. E. 2001, ApJ, in press (astro-ph/0103256)

Wilson, C. D., Scoville, N., Madden, S. C., \& Charmandaris, V. 2000, ApJ, 542, 120

\section{Discussion}

M. Fall: Isn't the simplest interpretation of your cluster and field star metallicity distributions for NGC 5128 that it results from the merger of two or more disk galaxies? Most of the field stars in the remnant elliptical galaxy would come from the progenitor disks, which would already have solved their G-dwarf problem in one way or another (giving metallicity distributions skewed to high metallcities). Furthermore, the globular clusters would have lower metallcities than the progenitor disk stars. Imagine the merger of a galaxy like the Milky Way with one like Andromeda. It would have just the kind of metallicity distri- 
bution you showed even without any later infall or accretion of dwarf galaxies. Isn't this the simplest explanation since Cen A does appear to be experiencing a merger of at least one gas-rich galaxy (in the recent past)?

W. Harris: I would guess there's probably a way to use the disk and thickdisk metallicity distributions of stars in disk galaxies to get the result you outlined (though I'm not sure offhand whether the globular cluster populations and metallicity distributions would come out right). We haven't looked into that quantitatively. However, on a basic level I'm not sure that approach is really simpler, since it shifts the same enrichment sequence problem back to the disk formation era. Instead, it seems simpler to us to use the "mergers" of large numbers of unenriched gas clouds which will be around in any case at high redshift.

B. Dirsch: Which AMR was assumed to transform the metallicity distribution of RGB stars into the number of all stars? You presented a graph with abundance increase vs. time. You mentioned that the model changed from a closed box model to an infall model while the time steps are always $\sim \Delta z$.

W. Harris: To start with, we have just assumed everything here to be "old" (i.e. no age-metallicity relation), which means all the color differences from starto-star is imagined to be due to metallicity. We realize, of course, that this is just a first step and not a substitute for a full CMD synthesis. The feature of the data which should make our first-order assumption OK is just that the RGB color spread is so large that it overwhelms small age differences. The model starts out with lots of gas infall, then dies away and gradually therefore converges to classic closed-box.

C. Grillmair: The use of $\log N$ vs. $\log M$ de-emphasizes differences between cluster systems (i.e. $4038 / 39$ vs. M87), vis-a-vis $\log N$ vs. magnitude.

$W$. Harris: Yes, $d N / d M$ emphasizes any common power-law shape while deemphasizing differences in mean mass. My point was really that $d N / d(\log M)$ isn't really Gaussian in shape.

R. Kennicutt: The extended timescale for star formation in your model may be inconsistent with observations of high-redshift galaxies, which suggest that most of the star formation in massive ellipticals was completed by $z \geq 3-5$. However, I believe that the metallicity distributions predicted in your chemical evolution models are scale-free in time. So is it possible that your model applies but the timescale is a few times faster?

W. Harris: Yes, that's quite possible. Within rough limits, we would imagine that a major formation period anywhere from 1 to 5 Gyr would work. Both the "stretch factor" from composition $z$ to time $t$, and the starting redshift $z_{0}$, are pretty flexible. If the $\Delta t$ gets too dramatically short then the star formation rate would get implausibly large, but if it stretches past $5 \mathrm{Gyr}$ or so, then younger stars would start showing up in the CMD. 